- Artículo original de investigación •

\author{
elocation-id: eia.n2a04
}




\title{
Efectos del monotributo en los pequeños comerciantes de los barrios Minitas, La Cumbre y Viveros de la ciudad de Manizales
}

\author{
Effects of the single-tax system on the small \\ retailers of Minitas, La Cumbre and Viveros \\ neighborhoods in the city of Manizales
}

Diana Fernanda Murillo-Aguirre', Claudia Viviana Salazar-Rodríguez², Hilda Yoana Zamora-Trujillo

Especialistas en Legislación Tributaria Nacional e Internacional, Facultad de Ciencias Contables, Corporación Universitaria Remington, Manizales Caldas, Colombia.

1 dianaf1978@yahoo.com

2 claudivisa@hotmail.com

3

Correo electrónico:

yoana1981zamora@gmail.com

\section{Resumen}

En virtud de poder contrarrestar los efectos del desempleo, la informalidad y las escasas oportunidades de tener una vejez digna en los estratos más vulnerables de la población, a través de la adopción de distintas políticas suscitadas al respecto en otros países de Latinoamérica, Colombia, con la reforma tributaria implementada mediante la Ley 1819 de 2016, buscó simplificar el proceso para el cumplimiento en las cargas tributarias en procura de lograr la formalización de los pequeños comerciantes, por medio del denominado monotributo, como mecanismo voluntario de recaudo que piensa convertirse en una alternativa del impuesto de renta y complementarios para las personas naturales cuyos ingresos tengan origen en actividades de comercio al por menor o peluquerías, disminuyendo en tal sentido la informalidad empresarial en el país. Sin embargo, según el reporte de responsabilidades Rut consolidado por la Subdirección de Gestión de Asistencia al Cliente de la DIAN, autoridad encargada de administrar el tributo en Colombia, durante el primer semestre del año 2017, fue evidente que la política del Gobierno tuvo mínima acogida, al parecer por la poca difusión y capacitación que al respecto impartió la administración de impuestos nacionales. En tal sentido, la mayor parte de la población entrevistada, comprendida por los comerciantes de los barrios La Cumbre, Viveros y Minitas, no contemplan en un corto plazo inscribirse en el monotributo de manera voluntaria, principalmente por las cargas tributarias onerosas que en el momento poseen y por falta de información sobre el mismo impuesto y los beneficios asociados a este nuevo tributo opcional. 
Palabras claves: beneficios, informalidad, impuesto, mecanismo, reforma tributaria, voluntario.

\section{Abstract}

Aiming to counteract the effects of unemployment, informality and limited opportunities for a dignified old age among the most vulnerable socioeconomic populations, Colombia, by adopting various policies implemented in other Latin American countries, enacted a tax reform law in 2016 (Act 819) that intended to make compliance with tax obligations easier for small retailers as well as to achieve formalization of small businesses. To this effect, a single-tax system (the so-called monotributo) was implemented as a voluntary collection mechanism that was expected to become an alternative income tax for individuals whose income derives from retail trade activities or from hairdressing and thus, reduce the business informality in the country. However, according to the RUT financial responsibilities report prepared by the DIAN's Customer Assistance Management Division, the agency in charge of tax enforcement in Colombia, during the first semester of 2017, the government's policy was scarcely adopted. This is arguably explained by the lack of promotion and training provided by the directorate of national taxes. Most of the population interviewed for this study, composed of small retailers of $\mathrm{La}$ Cumbre, Viveros and Minitas neighborhoods, do not foresee in the short time to voluntarily register for the single-tax system, mainly due to their onerous tax burdens and also to the lack of information about this new tax system and its benefits.

Keywords: benefits, informality, tax, mechanism, tax reform, voluntary.

\section{Introducción}

Las diferentes crisis económicas y sociales padecidas por los países latinos, auspiciaron el deterioro de la calidad de vida de las personas, originando el incremento del desempleo $y_{\text {, }}$ como consecuencia, la informalidad laboral; tal situación propició la formulación e implementación de regímenes especiales de tributación $y / o$ leyes como el monotributo, ajustados a las necesidades de un grupo objetivo, con características propias, en procura de legalizar a los trabajadores independientes, ofreciendo acceso a prestaciones laborales básicas, como cubrimiento en caso de accidente o enfermedades laborales, contar con una subvención para la vejez, aplicar a servicios financieros, y permitir contar con una reserva monetaria.

Partiendo de lo establecido en el artículo 2 de la Constitución Política de Colombia, donde está definido que el Estado tiene como fin esencial promover la prosperidad general, la cual puede tener como uno de sus pilares, la implementación de una ley tributaria que involucre regímenes simplificados que fomenten la actividad empresarial de forma clara y eficiente, se refleja que el Gobierno no ha logrado dicho objetivo, y se ha enfocado en aumentar el recaudo sin tomar en cuenta el bienestar económico y social de los contribuyentes.

El Estado ha procurado, mediante las diferentes reformas tributarias, subsanar falencias y situaciones que afectan la economía de la nación, y con la última reforma, promulgada por la Ley 1819 de 2016, en uno de sus apartes, hace mención a reducir la tasa de informalidad, que según la medición de empleo informal y seguridad social del trimestre abril-junio del DANE (2017), se ubica en $48 \%$, la cual se manifiesta en las altas cargas salariales, los costos operativos que implican constituir y/o liquidar empresa, y 
finalmente grava y dificulta el acceso y uso de productos bancarios para gran parte de la población, limitando las fuentes de financiación que buscan procurar capital de trabajo en los sectores productivos y comerciales.

En este mismo sentido, el aumento de la informalidad representaba para el Estado un importante foco de evasión tributaria, el cual no era más que el reflejo de un sistema tributario complejo, con muchos formalismos y con pocos beneficios para los comerciantes. Las crisis económicas ocurridas en los países latinos fueron el punto de partida que los obligó a fomentar leyes que creen alternativas de recaudación como el monotributo, en procura de legalizar a los trabajadores independientes, sin embargo, al inicio no se permitió la adhesión de un número significativo del grupo objetivo.

En la breve observación que se realiza en América Latina a la problemática de la informalidad, se hace evidente la preocupación y toma de medidas de varios países para la estructuración y ejecución de políticas públicas que contribuyan a la disminución de esta problemática social, originándose el sistema de monotributación para el sector de personas de bajos ingresos económicos, con el cual países como Brasil, Perú, Argentina y Uruguay han asumido el riesgo de hacerlo.

La mayoría de los países descritos anteriormente, cuentan con sistemas de tributación diferencial para los pequeños contribuyentes. Al observar a Perú, Argentina y Brasil se evidencia que su aplicación se realizó en los años noventa con el objetivo principal de lograr la formalización y un enfoque importante en las políticas sociales y económicas; este monotributo se identificó por simplificar los parámetros de registro, la presentación de las obligaciones con sus respectivas declaraciones y el pago de los tributos, así como vincular contribuciones dirigidas a la seguridad social.

De esta forma los gobiernos latinoamericanos han llevado a cabo políticas económicas tendientes a solucionar los problemas sociales y de empleo presentes en cada país. Así, entre los años 2000 a 2009 se notó una mejoría en las tasas de ocupación y de desempleo en la región. Por ejemplo, para el año de 2005, la tasa de desempleo y de ocupación se ubicó en el 9 \% y cerca del $54 \%$ respectivamente en toda América Latina (Ortiz, 2016, p. 2).

En relación a lo anterior, en la gran mayoría de países de América Latina han intentado equilibrar las consecuencias del desempleo, la informalidad, la ausencia de educación y las mínimas oportunidades de alcanzar una vejez digna en los estratos más vulnerables de la población. Es así como independiente del tipo de gobierno, las personas con el único propósito de subsistir y optimizar su calidad de vida, optan por todo tipo de actividades comerciales que les permitan satisfacer sus necesidades básicas, siendo este el principal efecto para el surgimiento de la informalidad y la falta de protección social en los denominados pequeños contribuyentes.

El Gobierno colombiano basándose en la experiencia de otros países de Latinoamérica, $y$ en los efectos obtenidos, buscando contrarrestar lo previamente referido, implementó el monotributo, en el artículo 165 de la Ley 1819 de 2016, como alternativa para disminuir dicha práctica y procurar que las personas informales, pudiesen hacerse contribuyentes activos del impuesto de renta.

Por lo anterior, a dieciocho meses de la entrada en vigencia de esta reforma, cuyo plazo máximo de inscripción fue el 31 de agosto del año 
2017, y según el reporte de responsabilidades Rut consolidado por la subdirección de gestión de asistencia al cliente de la DIAN fechado en julio 20 de 2017, hasta ese momento solo se habían efectuado 58 registros con esta obligación, de los cuales uno de ellos corresponde a la seccional de Manizales; se considera de suma importancia saber por qué esta estrategia del Gobierno nacional tuvo tan poca acogida, si se tiene en cuenta que la cantidad de personas del municipio de Manizales susceptibles de pagar dicho impuesto corresponde a 6860 microempresarios, distribuidos de la siguiente manera: 520 peluquerías pertenecientes a la actividad 9602 y 6340 comerciantes que cuentan con la actividad económica 47, según estadística consultada hasta el 1 de agosto de 2017 a través del registro único empresarial y social de las Cámaras de Comercio.

Esta investigación buscó resolver la pregunta: ¿Cuáles son los efectos del monotributo en los pequeños comerciantes de los barrios Minitas, La Cumbre y Viveros de la ciudad de Manizales?

\section{Materiales y métodos}

\section{Acerca del enfoque}

La investigación se realizó bajo un enfoque cualitativo y permitió establecer en un ambiente natural los motivos de la baja acogida que tuvo en su etapa inicial esta nueva estrategia gubernamental, y comprender la forma en que los microempresarios perciben su realidad frente a este nuevo sistema de recaudo, toda vez que se hace importante identificar las falencias en la implementación del monotributo. En este sentido, el enfoque cualitativo es recomendable para la situación problemática planteada, ya que el monotributo ha sido poco explorado en la población de trabajadores informales que se pretende impactar.

Por lo anterior, se aplicó el método no probabilístico de muestreo intencional, debido a que la población objeto es demasiado grande, asimismo, para su selección se consideraron algunas condiciones señaladas en el artículo 905 del Estatuto Tributo, que define a los sujetos pasivos del citado impuesto. Adicionalmente, se determinó escoger como grupo objetivo los barrios Minitas, Viveros y La Cumbre ubicados en la ciudad de Manizales, donde se encuentran microempresas que cumplen las características para aplicar al monotributo, con el fin de hallar respuestas a la pregunta de investigación, mediante la aplicación del instrumento de entrevista directa.

\section{Momentos de la investigación}

La investigación cualitativa tuvo cinco momentos metodológicos: revisión bibliográfica y reconocimiento de antecedentes, definición de las categorías del marco teórico, elaboración del instrumento de investigación, trabajo de campo y aplicación del instrumento $y$, por último, el procesamiento de la información.

\section{Revisión bibliográfica y reconocimiento de antecedentes}

El objetivo de este punto metodológico fue identificar las investigaciones realizadas sobre los inicios del monotributo, conocer los países pioneros en la implementación de este régimen y las diferentes estrategias del Estado para su masificación. También, la revisión bibliográfica permitió conocer los inconvenientes subyacentes a la ejecución de dicho régimen, tanto para los comerciantes (por falta de conocimiento 
sobre los temas tributarios) como para los diferentes Gobiernos referenciados, en razón de las múltiples categorías y requerimientos exigidos para su aplicación.

\section{Definición de las categorías del marco teórico}

Teniendo como base la poca o nula acogida que tuvo en su etapa inicial el nuevo régimen tributario denominado monotributo, a través de la presente investigación se pretendió identificar las falencias de su implementación, mediante la selección de la unidad de análisis determinada por los comerciantes de los barrios La Cumbre, Minitas y Viveros y por medio del reconocimiento de sus experiencias, creencias y perspectivas sobre el nuevo tributo opcional, con la ayuda de la aplicación de una entrevista en su contexto natural.

Es importante resaltar que del total de la unidad de análisis 130 comerciantes, se tomaron como unidad de trabajo para la investigación 40 de estos, los cuales fueron seleccionados de forma intencional con el fin de impactar diversas actividades económicas que permitieran la obtención de información variada, de interés para el planteamiento problemático y con la capacidad de generar resultados efectivos para el logro de los objetivos propuestos.

\section{Elaboración del instrumento}

\section{de investigación}

La investigación se realizó mediante un enfoque cualitativo de muestreo intencional, dado el interés por conocer desde el propio lenguaje y perspectiva de los comerciantes de los barrios La Cumbre, Minitas y Viveros, la realidad sobre la poca o nula acogida del impuesto voluntario, así como también las fallas en su implementación, teniendo en cuenta los beneficios aplicados al monotributo.

En este sentido, se hizo necesario la construcción de un instrumento investigativo basado en el enfoque metodológico de la investigación. Se eligió la entrevista directa con 20 preguntas estructuradas de acuerdo a los objetivos planteados de la siguiente manera:

Datos generales y principales características de la población. La entrevista inicia con los datos generales sobre el nombre, la actividad económica y la ubicación del establecimiento (La Cumbre, Minitas o Viveros) donde se encuentra ubicado el establecimiento de comercio. Luego, las preguntas correspondientes a los numerales 1 al 8 identifican si el establecimiento se encuentra legalmente registrado y qué tipo de afiliaciones maneja el comerciante en materia de salud, pensión, riesgos profesionales y caja de compensación; lo cual es indispensable para una posible aplicación de los beneficios del monotributo.

Identificación de las percepciones de los pequeños comerciantes de los barrios $\mathrm{La}$ Cumbre, Minitas y Viveros sobre el monotributo. Por medio de 7 preguntas de los numerales 9 al 14, se indagó sobre el conocimiento de los comerciantes en cuanto a los temas tributarios y particularmente sobre el monotributo, resaltando el interés o la falta de interés sobre este nuevo régimen y evidenciando el impacto de las estrategias del Gobierno para su difusión y/o masificación.

Identificación de las falencias y percepciones de la realidad. En las restantes 5 preguntas comprendidas en los numerales 15 al 20 de la entrevista aplicada a los comerciantes, se buscó comprender a través de vivencias desde un ambiente real, 
las fallas en materia de difusión, las diversas actitudes o comportamientos asumidos por los comerciantes ante todos los temas tributarios $y$, finalmente, identificar la posible aplicación de un beneficio asociado al monotributo desde la propia necesidad del comerciante.

\section{Trabajo de campo, aplicación del instrumento}

Teniendo como base la unidad de análisis de los comerciantes de los barrios La Cumbre, Minitas y Viveros y la unidad de trabajo de 40 comerciantes, se procedió a seleccionar dos zonas estratégicas para la aplicación del instrumento, cada una de ellas con 20 establecimientos, donde la zona 1 (La Cumbre) impactó la mayor área de afluencia de establecimientos y la Zona 2 (Minitas y Viveros) identificó gran variedad de actividades económicas.

\section{Procesamiento de la información}

Posterior a la aplicación del instrumento, el procesamiento de la información se realizó a través de gráficas estadísticas para algunas de las respuestas de connotación cuantitativa y para las respuestas de tipo cualitativa se realizó análisis de contenido a cada una de las expresiones, vivencias, creencias, quejas, sugerencias y todo tipo de información que permitiera el reconocimiento de las estrategias del Gobierno y su impacto directo sobre los denominados pequeños comerciantes y principales sujetos pasivos del monotributo.

\section{Resultados}

Es claro que el interés principal del Estado es lograr la formalización de las economías generadoras de capital, para posteriormente asegurar el incremento en el ingreso de los tributos; bajo esta misma línea se sustentan sus políticas de incentivar el cumplimiento voluntario de las obligaciones formales en términos de legalidad y oportunidad, facilitando los procesos y la gestión de trámites y servicios, enfocando estrategias y acciones de control según las características de cada segmento (comercial, industrial y de servicios y por estratos socioeconómicos), para optimizar la gestión de cobro de impuestos $y$, finalmente, incrementar el recaudo de los mismos; el ideal es hacer que este comportamiento sea creciente a largo plazo y, por ende, reducir la brecha fiscal.

Pero, más que los niveles de pobreza, la falta de educación tributaria, las precarias condiciones laborales, todo esto aunado a la actual situación que vive el país con respecto a las malas acciones de sus dirigentes y autoridades, requieren un esfuerzo extraordinario que converja en el mejoramiento de la gestión y la creación de un ambiente positivo que aumente la confianza en la administración tributaria.

La visión de la Dirección de Impuestos y Aduanas Nacionales de Colombia, definida en el plan estratégico DIAN 2014-2018, procura generar un alto nivel de cumplimiento voluntario de las obligaciones tributarias, aduaneras y cambiarias, apoyar la sostenibilidad financiera del país y fomentar la competitividad de la 
economía nacional, gestionando la calidad y aplicando las mejores prácticas internacionales (Galvis, 2015). Por tanto, los enfoques hacia el servicio, la fiscalización y el control, son los pilares de una gestión eficaz, la cual no es posible sin el conocimiento estructurado de las necesidades de los contribuyentes y su apoyo en la meta de recaudo.

Partiendo de la base que el sujeto pasivo es el obligado a cumplir, sea en calidad de contribuyente o de responsable con las exigencias tributarias, tal como lo manifiesta el Estatuto Tributario (2016) en su artículo 792, y en aplicación de lo establecido en el artículo 165 de la Ley 1819 de 2016, es importante señalar las condiciones que se deben cumplir para pertenecer al monotributo, las cuales son:

1. Que en el año gravable hubieren obtenido ingresos brutos ordinarios o extraordinarios, iguales o superiores a 1400 UVT e inferiores a 3500 UVT.

2. Que desarrollen su actividad económica en un establecimiento con un área inferior o igual a 50 metros cuadrados.

3. Que sean elegibles para pertenecer al Servicio Social Complementario de Beneficios Económicos Periódicos, BEPS, de acuerdo con la verificación que para tal efecto haga el administrador de dicho servicio social complementario.

4. Que tengan como actividad económica una o más de las incluidas en la división 47 comercio al por menor y la actividad 9602 peluquería y otros tratamientos de belleza de la Clasificación de Actividades Económicas Revisión 4 adaptada para Colombia CIIU Rev. 4 A.C. Adoptada por la Dirección de Impuestos y Aduanas Nacionales (p. 84)

Para la correcta selección de la muestra o subgrupo de población de interés de la investigación, se partió de una base de datos suministrada por la Cámara de Comercio de Manizales por Caldas, que contenía la información de los comerciantes inscritos con las actividades económicas que califican, según el numeral 4 del artículo 905 del Estatuto Tributario, ya mencionado, para acceder al monotributo, ubicados en el sector de interés, y que una vez segmentada en los barrios objeto de estudio, arrojó un total de 130 individuos. En tal virtud, se decidió aplicar la entrevista al 31 \% de dicha población dando como resultado un total de 40 posibles sujetos de estudio.

Con respecto a los numerales del artículo referido, es preciso aclarar, que la norma hace referencia al año gravable 2017 y el rango de ingresos está comprendido entre $\$ 44,602,600$ y $\$ 111,506,500$; que la verificación para determinar quiénes son elegibles para pertenecer al servicio social complementario de beneficios económicos periódicos (BEPS), compete a Colpensiones, según lo establece el artículo 155 de la Ley 1151 de 2007 y el artículo 17 del Decreto 604 de 2013; que las actividades correspondientes a la división 47 de comercio al por menor, señaladas en la Resolución 139 de 2012, por la cual la DIAN asume la Clasificación de Actividades Económicas - CIIU revisión 4 ajustada al territorio nacional, son 31 actividades económicas y adicionando el código 9602, da un total de 32 actividades económicas aplicables para el monotributo; finalmente, que mediante Decreto 939 de 2017, en el Inciso 8 de la parte considerativa se aclara que la expresión «106» es un error caligráfico, que no incide en lo ordenado por el estatuto.

La comuna ecoturística Cerro de Oro de Manizales, es un sector conformado por 10 barrios, entre los que se encuentran La Cumbre, Viveros y Minitas, ubicados en el estrato 3 , calificado mediante el Acuerdo 589 de 2004, 
que, según el Departamento de Planeación Nacional, corresponde al nivel medio-bajo, y se seleccionaron para el estudio de interés, debido al alto número de establecimientos de comercio que se enmarcan dentro de las características del impuesto voluntario, y en los cuales se aplicaron 9, 13 y 18 entrevistas respectivamente, a criterio del entrevistador.

Adicionalmente, a continuación, se listan los establecimientos visitados, por actividad económica, según la clasificación del CIIU Rev. 4 A.C. (clasificación industrial internacional uniforme de todas las actividades económicas Revisión 4 adaptada para Colombia CIIU Rev. 4 A.C.), que es la clasificación internacional que reseña las actividades económicas productivas (Tabla 1):

Tabla 1. Adaptada de CIIU Rev. 4 A.C. DANE (2012) p. 67-68.

\begin{tabular}{|l|c|c|}
\hline \multicolumn{1}{|c|}{ Tipo de establecimiento } & $\begin{array}{c}\text { Actividad } \\
\text { económica }\end{array}$ & Total \\
\hline Tiendas & 4711 & 14 \\
\hline Peluquerías y barberías & 9602 & 9 \\
\hline Papelerías & 4761 & 3 \\
\hline Reverterías & 4721 & 3 \\
\hline Droguerías & 4773 & 3 \\
\hline Venta de artículos electrónicos & 4772 & 2 \\
\hline $\begin{array}{l}\text { Venta de artículos de cuero } \\
\text { y zapatería }\end{array}$ & 4741 & 1 \\
\hline Carnicerías & 4723 & 1 \\
\hline Cacharrerías & 4712 & 1 \\
\hline Venta de prendas de vestir & 4771 & 1 \\
\hline Avícola y quesera & 4722 & 1 \\
\hline Caseta & 4724 & 1 \\
\hline
\end{tabular}

Teniendo en cuenta que la formalización de un establecimiento de comercio implica, comúnmente, la obtención del certificado de existencia y representación legal o matrícula mercantil, el uso de suelos, inscripción en el RUT, registro de libros contables, en caso de estar obligado y siendo los más comunes libro diario, libro mayor y balances, inventarios, libro de actas, registro de socios, registro de asambleas y de juntas, cuya obligación depende del tipo de persona (natural o jurídica) y la responsabilidad en el régimen de ventas (simplificado o común), y registro ante el Invima, exigido para los expendios de medicamentos, entre otros; durante la realización del estudio se pudo determinar que el $70 \%$ de los entrevistados cuenta con registro mercantil, RUT, industria y comercio, registro sanitario y permiso de bomberos, el $10 \%$ posee RUT y matricula mercantil, y en igual proporción solamente un pequeño grupo cuenta con RUT, esto último, debido a que el trámite no representa ningún costo para el comerciante; de esta unidad de trabajo, solamente 2 personas, correspondientes al $5 \%$ de la muestra, no cuentan con ningún registro, debido a que no tienen un aviso que evidencie al público la realización de la actividad comercial. Para la mayoría, la formalización implica considerables costos en el funcionamiento de su negocio, y en general, los requisitos implican pago de tarifas que pueden ser fijas u oscilar según el capital o el tipo de actividad que se realiza. Se coincide en que la legalidad no incide en la rentabilidad de la actividad mercantil. Por lo anterior, no evidencian ningún tipo de rentabilidad o ahorro en el pago de impuestos, creen que no existen las subvenciones y que el acceso a financiación implica adquirir deudas que redundan en un alto precio a la hora de amortizarlas.

En cuanto a la periodicidad en la oportunidad de la renovación de los anteriores registros, el $85 \%$ de los participantes manifestó que cumple con esta obligación de forma anual, la cual es expresa para la mayoría de los mismos, sin embargo, es de aclarar que la obligatoriedad en 
la actualización del Registro Único Tributario (RUT) nace cuando se presentan cambios en la información allí contenida, de otra forma la administración tributaria, en el Decreto 2460 de 2013, expresa: «Sin perjuicio de las actualizaciones a que haya lugar, la inscripción en el Registro Único Tributario (RUT), tendrá vigencia indefinida y, en consecuencia, no se exigirá su renovación» (Art. 6).

En igual proporción al porcentaje anterior, 34 entrevistados señalan pertenecer al régimen simplificado de las ventas, y el resto manifestó no pertenecer a ninguno de estos sistemas; adicionalmente, con los artículos 1, 5, 4 y 8 del Título 4 del Decreto 738 de 2017, el Gobierno nacional estableció que las responsabilidades de ventas régimen común, renta y el monotributo fueran excluyentes, al señalar que si el monotributista incumple alguno de los señalamientos del artículo 499 del Estatuto Tributario, en el período gravable o a su cierre, este deberá actualizar el RUT, registrando las responsabilidades propias del régimen común del impuesto sobre las ventas y del régimen del impuesto sobre la renta y complementarios.

El $42.5 \%$ de la unidad de trabajo afirma que su sustento depende exclusivamente del establecimiento de comercio, en tal sentido se vislumbra que, debido a la necesidad de incrementar los ingresos, en promedio el propietario trabaja entre 12 y 14 horas por día, creando con esto la obligación de que varias personas del núcleo familiar participen de la labor comercial, trabajando por turnos, o en menor proporción están los que procuran tiempos de descanso partiendo la jornada laboral en dos. El $57.5 \%$ restante, cuentan con la posibilidad de complementar los ingresos del grupo familiar porque alguno de sus miembros es asalariado, pensionado, practican la agricultura o trabajan en transporte público, entre otras actividades económicas.
Respecto a la afiliación al régimen de salud, se percibe que algunos de los entrevistados no tiene conocimiento que en Colombia se cuenta con dos sistemas: el subsidiado, que cobija a la población de bajos o nulos ingresos económicos y que no tienen capacidad de pago, permitiendo por medio de las subvenciones que ofrece el gobierno, tener acceso a servicios de salud; y el contributivo, al cual se afilian las personas que tienen ingresos por contrato laboral, de prestación de servicios, los trabajadores formales e independientes, los pensionados, en todo caso, quienes tienen liquidez. En consecuencia, no tienen clara la diferencia entre los mismos.

El estudio señala que el $47.5 \%$ pertenece al régimen subsidiado, y en este punto cabe aclarar que, según el Ministerio de Salud, a este orden no se adhieren las personas que aplican para el régimen contributivo, previamente enunciado; asimismo, se identifica de forma expresa como favorecidos a los desmovilizados, indígenas, habitantes de la calle, niños en protección y desplazados; y en menor proporción con un $32.5 \%$, se encuentran los del sistema contributivo y el resto, correspondiente a 8 personas, quienes no cuentan con ninguna protección en salud, afirmando en la mayoría de los casos que para poder acceder al cubrimiento en salud, las entidades les exigen afiliarse conjuntamente a pensión, y que no tiene los ingresos suficientes para cubrir ambos beneficios y seguir respondiendo por las obligaciones propias del sostenimiento familiar y del negocio; adicionalmente, es generalizada la creencia que las personas jóvenes y de mediana edad no tendrán derecho al disfrute de su mesada a causa de la brecha y la deuda pensional.

Continuando con la pertenencia al Sistema General de Pensiones, el estudio reporta que del total de la muestra, el $67 \%$ no se encuentran 
afiliados al mismo, indicando como justificación a tal situación, no contar con la solvencia económica suficiente para acceder a este beneficio, máxime si la Ley 100 (1993), señaló en forma obligatoria a «los trabajadores independientes con capacidad de pago" (Art.15) y en la mayoría de los casos los participantes aducen no contar con recursos económicos para subsidiar tal obligación; asimismo, una porción menor de este porcentaje no aportaba a tal régimen puesto que ya disfrutaban de su mesada pensional; finalmente, el equivalente a un $30 \%$, se encuentra aportando al sistema pensional, en tal sentido, menos de la tercera parte de los entrevistados cumple con la referida contribución.

En comparación con la inscripción al sistema general de riesgos profesionales laborales, el $87.5 \%$ de los indagados, manifestaron no estar incorporados en dicha modalidad, principalmente porque la equiparan con el régimen de salud, creencia que existe, puesto que no hay claridad respecto de la función de dicho sistema, que está encargado de prevenir y asistir a los trabajadores en caso de enfermedad o accidente que se derive de la realización de la labor que desarrollan. Adicionalmente, la Ley 1562 de 2012 «por la cual se modifica el sistema de riesgos laborales y se dictan otras disposiciones en materia de salud ocupacional» estableció que los trabajadores independientes e informales pueden afiliarse de forma voluntaria a este régimen; sin embargo, el $12.5 \%$ sobrante, sí considera importante participar de los beneficios que ofrece esta alternativa de protección.

Según lo establecido en el artículo 19 de la Ley 789 de 2002, los trabajadores independientes podrán incorporarse libremente a las cajas de compensación familiar, las cuales les permitirán a este y a su núcleo familiar, participar de beneficios como salud, educación, recreación, cultura, turismo, deporte, vivienda, crédito y microcrédito. Al respecto, el $90 \%$ de los entrevistados indicó no estar afiliados a este tipo de entidades, puesto que el aporte correspondiente por adherirse depende de la base de cotización con la que se realizan los aportes al sistema de salud y pensión, y solamente el $10 \%$ se encuentran vinculados a algún tipo de caja de compensación.

Por lo anterior, es de precisar que, para acceder al sistema general de riesgos profesionales laborales, se exige previo a su inscripción, estar debidamente afiliado al régimen de salud, y para acceder a los beneficios de las cajas de compensación familiar; adicionalmente, se debe inscribir al régimen pensional, para efectos de liquidar la respectiva base, obligando con esto, destinar un alto volumen de las ganancias obtenidas al cubrimiento de estas responsabilidades.

\section{Percepciones de los pequeños} comerciantes sobre el monotributo como impuesto voluntario

El recaudo de los impuestos en todos los países cobra gran importancia como la fuente económica para cumplir con todas las obligaciones del Estado, pues es este, quien debe garantizar el bienestar de los ciudadanos, y es aquí donde se aplica el artículo 95 de la Constitución Política que indica: «contribuir al financiamiento de los gastos e inversiones del Estado dentro de los parámetros de justicia y equidad» (numeral 9), es así, como todos los ciudadanos cuentan con la obligación de coadyuvar de forma proporcional y equitativa por medio de los impuestos al sostenimiento del Estado. 
A través de la historia tributaria, Colombia ha atravesado por crisis macroeconómicas que han conllevado a reestructurar los marcos normativos, pensando no solo en el fortalecimiento fiscal a través del aumento de los ingresos mediante los impuestos, sino también en favorecer los diferentes sectores de la economía tratando de no ser tan drástico con las utilidades de las personas jurídicas y personas naturales; de allí la razón de la Ley 1429 de 2010 que estimuló el empleo formal, la Ley 1607 de 2012 cuyos objetivos fueron mejorar la competitividad de las empresas, en especial aquellas que recurrieran a la mano de obra intensiva, optimización de la carga tributaria y que posibilitaran la inclusión de la población vulnerable a la economía formal.

Tristemente, a la fecha lo único que se ha conseguido es agudizar un problema de cumplimiento en cuanto a los deberes formales de los contribuyentes y aumentar la ineficacia del sistema tributario, con la ley 1819 de 2016 se buscó mejorar las condiciones de los pequeños comerciantes, incentivando la legalización de los negocios y generando calidad para competir, a través del nuevo impuesto voluntario denominado monotributo.

Revisando el contexto real de los comerciantes, en el trabajo de campo realizado, los entrevistados manifestaron no tener claridades sobre el concepto de qué es un impuesto, debido a la falta de una cultura tributaria la cual es conceptualizada por Rojas (2015) como los «valores, creencias, saberes y comportamientos, resultantes y condicionantes de la interacción entre la contribución de la sociedad y el servicio ofrecido por el Estado, cuyo propósito es la construcción y desarrollo de la sociedad" (p.19), este por tanto no es un concepto con el que el ciudadano del común se encuentre identificado, puesto que algunas de sus respuestas con respecto a qué es un impuesto fueron: «Lo que cobra el Gobierno por la prestación de servicios públicos, todo lo que impone el gobierno para su propio interés, es algo que se paga, es algo que uno paga para poder trabajar, es un porcentaje que se le paga al Gobierno por tener lo suyo, una deuda más, lo más maluco que puede haber»; lo que permite comprender que los entrevistados no vislumbran la razón de ser de los impuestos, sus efectos y contribuciones para el país y su implicación es el bajo cumplimiento de las obligaciones con el Estado.

Asimismo, la cultura tributaria no es responsabilidad de unos pocos ciudadanos, es el cambio de conciencia de providencia constitucional que obliga al ciudadano a contribuir, como lo refiere Vega (2015) que la raíz de esta problemática es que en el país no hay cultura tributaria. La evasión de impuestos es general en Colombia, no discrimina estrato ni afiliaciones políticas. Sin embargo, es claro que la falta de recaudo entre los segmentos de mayor ingreso representa un daño mayor, pues el monto que se ha dejado de recibir por el impuesto a la renta asciende a casi $\$ 28$ billones. Es pues necesario que se realice pedagogía en cuanto a cultura tributaria, se debe formar en todos los niveles de la sociedad, sin exclusiones.

Dado lo anterior, se hizo necesario formular la pregunta: ¿usted sabe para qué sirven los impuestos? Los entrevistados en su gran mayoría tuvieron una reacción inmediata, con respuestas que se relacionaban con la mentalidad generalizada de la corrupción y malversación de los recursos, pero sí identificaban, en alguna proporción, el uso de los impuestos para la inversión social (educación, salud, vivienda), manifestaron su vivencia del desempleo y pobreza generalizada, ya que por su actividad mercantil los ciudadanos compran el diario y aducen que la solución no está en el aumento de tributos, 
ni en la excusa de evitar la evasión; sino en la presencia institucional, en el manejo de políticas que garanticen seguridad y confianza, en el diseño de criterios económicos que redunden en un bienestar social más no en una afectación del bolsillo del ciudadano de a pie, y así gestar en el tiempo una economía sólida y de progreso.

Es así como los estudios realizados por el Estado no son socializados y en su mayoría no profundizan en la capacidad contributiva de un ciudadano de estrato 1 y 2 al reformar impuestos como el IVA, que establece una tarifa sin determinar la realidad económica de un país que vive en la informalidad, sin estudiar los ingresos reales de las personas que luchan por su supervivencia y se genera una percepción donde el colombiano reacciona a la necesidad de huir del tributo, porque representa una carga, llegando a considerarlo no como un impuesto sino como una sanción. Aunque la razón del impuesto no es sancionatoria, en el pensamiento colectivo se pierde la esencia misma del tributo, y es aquí donde el ciudadano piensa que sus ingresos y el patrimonio no se afectan si se aprende a evadir impuestos.

Por lo anterior, el Gobierno tiene como reto hacer entender al contribuyente la relación que existe entre el ciclo tributario y la distribución que se hace con los ingresos que se perciben por los tributos, tratando de evitar los problemas que se pueden presentar como son la evasión, la elusión, el contrabando, el fraude fiscal y el no pago correcto de los tributos (Bonilla, 2014, p. 24).

Además, la falta de confianza en la disposición de estos recursos, donde a más impuestos más corrupción, lo vive el ciudadano de a pie cuando se relaciona con lo público y encuentra deficiencia o ausencia en el sistema de salud, proyectos de infraestructura convertidos en elefantes blancos y una justicia deficiente e ineficaz. Es importante reconocer aquí que el tributo tiene una función social, bajo los principios que constitucionalmente rigen en el sistema tributario, entre ellos están: la igualdad, la equidad, la justicia, la certeza, la progresividad y la legalidad.

Por lo tanto, es importante que la administración tributaria diseñe propuestas basadas en técnicas de motivación, donde se capacite con información clara y entendible a los pequeños comerciantes sobre sus deberes y responsabilidades, lo que conduciría a la formación de un compromiso social, que podría redundar en la equidad de la distribución de la carga tributaria, con la conciencia normativa de la obligación a contribuir y esto de algún modo reflejaría la reducción en la evasión.

A pesar de la desconfianza manifestada por los entrevistados, es importante resaltar que un $45 \%$ no sabe cuál es la entidad que administra los impuestos, lo que confirma nuevamente la ausencia de cultura tributaria, y un $35 \%$ reconoce a la DIAN como el ente administrador, los demás entrevistados optan por nombrar cualquier otra entidad pública, demostrando en su actitud la baja importancia que les representa conocer quién es el ente administrador.

Pero si analizamos los impuestos que conocen los entrevistados, un $25 \%$ mencionan algunos de carácter territorial entre ellos el predial, cámara de comercio, industria y comercio, valorización, entre otros; un $60 \%$ indican algunos impuestos de orden nacional, destacando el IVA como el impuesto más conocido y un $15 \%$ manifiestan no conocer el nombre de algún impuesto; por lo que se evidencia que el aporte realizado en la formalización de sus establecimientos de comercio no los consideran como un impuesto, sino como un valor a pagar para ejercer a actividad comercial. 
Considerando el Impuesto de Renta como uno de los más fáciles de identificar por su difusión amplia, la importancia que el Estado le brinda por su incidencia en la contribución a las arcas del Estado y el temor que la administración tributaria infunde de forma indirecta hacia el ciudadano por las multas y sanciones que acarrea el no pago de este impuesto, se convierte en una pregunta para la unidad de trabajo seleccionada, detectando que el $50 \%$ de los entrevistados no declara, un $20 \%$ declara renta, un $23 \%$ no declara, pero conoce los topes y un $7 \%$ considera que este impuesto es el que se paga cuando se posee propiedades.

Es así como se precisa que, dentro de la unidad de trabajo, un $20 \%$ podría optar por la inscripción en el monotributo; cuyo impuesto voluntario trata en su esencia de mostrarse simple y sencillo, como una opción que simplifica el pago del impuesto de renta y complementarios, a la vez como una elección beneficiosa que procura bajar los índices de informalidad y hacer más amigable el recaudo tributario, recibiendo los beneficios que promete este nuevo gravamen, entre ellos, el acceso a créditos para optimizar y consolidar la actividad mercantil, e impulsar el gran desafío de la formalidad, dando acceso al cumplimiento oportuno de los deberes tributarios de los contribuyentes que se acojan de forma voluntaria a dicho régimen.

El Banco Interamericano de Desarrollo, en su investigación realizada al sector económico de los pequeños comerciantes, señala que el monotributo es un tributo pequeño o un tributo único, y en países donde ya se aplica (Argentina, Perú, Brasil, Uruguay, Costa Rica, entre otros) a este sistema de tributación se le llama «Régimen Simplificado para Pequeños Contribuyentes» el cual consiste en un régimen impositivo integral, por medio del cual los sujetos pasivos cumplen con las obligaciones tributarias relativas a la renta, al IVA y al sistema de seguridad social (González, 2006). En primer lugar, se debe determinar un margen de pagos, relacionados en un estimativo de tarifas progresivas, según las actividades económicas de los monotributistas que revisan sus ingresos promedio; en segundo lugar, el punto donde se encuentran ubicados los establecimientos y las demás condiciones que se exijan en el país donde se implemente este tipo de contribución.

Adicional a lo anterior es importante tener en cuenta lo manifestado por la Organización Internacional del Trabajo (2014) haciendo referencia a que «la informalidad de las economías latinoamericanas está generando diversos efectos negativos sobre el mercado de trabajo, la protección social y la recaudación tributaria» (p. 65). Y concluye afirmando que este impuesto cumple con las características de un enfoque integral, convirtiéndose en un instrumento práctico que aporta a la recaudación tributaria.

Es importante revisar lo manifestado por el programa regional de empleo para América Latina y el Caribe (PREALC) de la organización mundial del trabajo, citado por Mérida (2014), que describe a la economía informal «como un sector económico que se desarrolla con base a la subsistencia familiar, por medio de actividades con un alto uso de mano de obra, tecnología relativamente atrasada, poca productividad y con ganancias reducidas» (p. 4). Aún para el año 2017 esta situación sigue siendo evidente y continúa sin alternativas claras y asequibles, que puedan influir de manera positiva en la economía, en la acentuación del desempleo que sigue creciendo, como también la tasa de informalidad que aumenta a razón de la migración de personas a las capitales, como consecuencia de la falta de demanda laboral. 
Algunos de los entrevistados manifiestan que la formalidad incrementa el costo tributario al asumir el pago de salarios con prestaciones conforme a la ley, además, indican que no tienen motivaciones para formalizarse, pues transitar al sector formal es una decisión económica que influye en las ganancias. Dado lo anterior fue preciso indagar en la siguiente pregunta ¿Conoce el nuevo tributo opcional denominado monotributo?, la respuesta en un $55 \%$ fue que no, lo que representa un impacto negativo en el aspecto social, debido a la desinformación de los comerciantes sobre los beneficios a los que pueden optar en caso de acogerse al monotributo; pero un $32 \%$ de la unidad de trabajo si tenía algún tipo de conocimiento sobre el impuesto, por lo informado en algunos medios de comunicación o por comentarios de algunos clientes, sin embargo, el conocimiento no es amplio, ni claro, ni preciso.

Al respecto, de acuerdo con lo manifestado por los entrevistados, se puede concluir algunos aspectos positivos del monotributo como las cuotas impositivas bajas, la forma de pago mensual en relación a los ingresos recibidos, el aporte al sistema de seguridad social en pensión, la posibilidad de contratar empleados sin generar sobre costos en la tributación; pero también se fundan algunos aspectos negativos como el nivel de recaudación mínimo por la falta de información y desconocimiento del gravamen, pues pocos contribuyentes se han postulado, y aún no existe un control por parte de la DIAN para administrarlo. La condición más relevante de los beneficiarios no debería estar enmarcado por los ingresos, sino que debería considerar otras variables; además, no se conoce la normatividad ni las condiciones para acceder al crédito que promete el Estado a través de entidades bancarias, y el limitante a un solo establecimiento genera una gran desventaja.
Los datos recolectados, en cuanto al desconocimiento de la existencia del Impuesto voluntario monotributo, hace necesario que el comerciante incurra en gastos al contratar una asesoría tributaria, siendo la única opción para obtener la información sobre el nuevo impuesto, a razón de la poca difusión en los medios de comunicación y por parte de la administración tributaria, en otorgarle la importancia que indica la reforma tributaria en el impacto que este impuesto promete en la evasión y elusión fiscal, pues las 22 personas entrevistadas que no conocen sobre el impuesto estarían interesadas en saber más de él, ya que la concepción de impuesto voluntario es ambiguo y alerta a los comerciantes, quienes se tranquilizan al escuchar que es voluntario y opcional.

Por lo tanto, este análisis de resultados muestra cómo este impuesto busca encontrar a los comerciantes informales, cuyos ingresos los convierten en sujeto pasivo del impuesto de renta, $y$ a raíz de su situación de informalidad siempre han evadido el pago de dicho impuesto. Es así como el Estado quiere encontrar el método o la fórmula eficaz para cobrar estos dineros, y adoptar un tributo con recorrido internacional, con la facilidad en su liquidación, que se muestre económico y brinde beneficios a los que solo acceden los ciudadanos que laboran de forma dependiente o los que de forma independiente pagan sus obligaciones para acceder a un trabajo

Aunque cada comerciante toma su decisión de manera voluntaria para dar el paso al monotributo, este lo hará de acuerdo a sus perspectivas, analizando las ventajas y desventajas en su aspecto económico y personal, pero sin la base de formación tributaria que lo haga entender la naturaleza de los impuestos, su destinación, importancia y control de los mismos. Se gesta 
entonces el reto del monotributo, en cabeza de la DIAN, entidad que debe motivar la formalización y determinar los beneficios reales con los que contarán los inscritos y la responsabilidad, por supuesto, de persuadir a los contribuyentes para el cumplimiento voluntario de las obligaciones formales e impositivas con un sistema que se muestra de bajo costo y sencillo.

\section{Discusión}

\section{Identificación de las falencias en la implementación del monotributo}

El Gobierno colombiano ha intentado a través de las distintas reformas tributarias contrarrestar los efectos del desempleo, la informalidad, la falta de educación y las pocas oportunidades de una vejez digna en los estratos más vulnerables de la población. Sin embargo, dicho esfuerzo resulta insuficiente en la medida que las personas con el único propósito de subsistir o mejorar su calidad de vida, optan por todo tipo de actividades comerciales que le permiten satisfacer sus necesidades básicas, siendo este el principal efecto para el surgimiento de la informalidad y la falta de protección social en los denominados pequeños contribuyentes.

La evasión tributaria en los contribuyentes más pequeños, en muchos casos fue alentada por la complejidad y excesivo formalismo del sistema tributario. Entonces era necesario un mecanismo que le permitiera a estos sujetos cumplir con sus obligaciones fiscales y previsionales de una manera más simple y acorde a sus posibilidades (Rodríguez, Chaumont y León, 2011, p. 32).

Mediante la Ley 1819 del 2016, el Estado da inicio a un régimen tributario que tiene como principal objetivo disminuir la informalidad y optimizar los recursos del Estado, pero para ello utiliza como base la imposibilidad de los pequeños contribuyentes para vincularse a un sistema de seguridad social, que por último es responsabilidad del Estado garantizarlo, al respecto se refieren Ballesteros, Herrera y Parra (2014):

Lo que es importante para el derecho laboral, es el intento del Estado Colombiano de suplir lo que concierne al Derecho de la Seguridad Social, en aquellas minorías que, por motivos laborales y económicos, no pueden hacerse cargo de estos, por lo tanto, no hay garantía del mínimo vital (p.11).

En este sentido y a partir del 1 de enero de 2017, empezó a regir el nuevo tributo opcional de determinación integral, con el cual el Gobierno pretende reducir las cargas formales y sustanciales, impulsar la formalidad y en general simplificar y facilitar el cumplimiento de la obligación tributaria de los contribuyentes que voluntariamente se acojan al nuevo régimen del monotributo según lo establecido en el artículo 165 de la ley 1819 del 2016. Asimismo, mediante dicho artículo se establece el valor a pagar por el monotributo, el cual dependerá de la categoría $(A, B, C)$ a la que pertenezca el contribuyente y este a su vez de sus ingresos brutos anuales. Adicionalmente, el monto pagado por concepto del monotributo tiene dos componentes, un impuesto de carácter nacional y un aporte al servicio Social Complementario BEPS o un aporte al Sistema General de Riesgos Laborales según el artículo 907 del que trata el libro VIII del Estatuto Tributario.

Luego de establecer la unidad de trabajo y de aplicar una metodología no probabilística, los microempresarios entrevistados contestaron no tener conocimiento del nuevo impuesto denominado monotributo, cuya principal causa 
puede ser la falta de difusión sobre el tema, pero llama la atención que una pequeña porción contestó que habían escuchado algo al respecto; sin embargo, los tres motivos principales para dicho desconocimiento es la indiferencia sobre los temas tributarios, el deseo de no pagar otro impuesto adicional y la poca o nula información sobre los beneficios que aplican al monotributo.

Cabe resaltar sobre estos últimos, que el $82 \%$ de la población objeto de estudio no conoce dichos beneficios y mucho menos su forma de operatividad dentro del recaudo; por su parte, el $18 \%$ de los microempresarios que conoce o ha escuchado hablar sobre los beneficios tienen una idea vaga al respecto o responden de acuerdo con sus propias necesidades, indicando que es aplicable a salud y pensión, lo cual quizás no esté tan ajustado a la realidad, pero si representa un fiel reflejo de las necesidades básicas insatisfechas por parte del Gobierno, no solo para los comerciantes de los barrios Minitas, La Cumbre y Viveros, sino también para todos los micro y pequeños empresarios del país.

Es importante resaltar al respecto de los beneficios asociados al monotributo al que se refiere la Ley 1819 (2016), que «podrán acceder al esquema de aseguramiento para riesgos de incapacidad, invalidez o muerte establecido para el servicio social complementario de los beneficios económicos periódicos (BEPS), cuya prima será asumida por el fondo de riesgos profesionales» (Art.168). Por su parte, la Ley 1819 (2016), establece que «los contribuyentes de que trata el párrafo 1 del Artículo 905 del Libro VIII del Estatuto Tributario podrán acceder al sistema general de riesgos laborales de que trata la ley 1562 de 2012, en los términos que define el Gobierno Nacional» (Art.169).
El tercer beneficio está contemplado en la Ley 1819 (2016) y define que:

Para los contribuyentes que hayan optado por el monotributo al que se refiere el Libro VIII del Estatuto Tributario, con excepción de los sujetos pasivos del que trata el párrafo 1 del artículo 905 de dicho Libro, la inscripción en el RUT como contribuyentes del monotributo generará automáticamente su vinculación en el servicio social complementario de los beneficios económicos periódicos (Art.170).

En complemento de este último esquema, los beneficios económicos periódicos (BEPS) constituyen un mecanismo eficaz para que un porcentaje amplio de la población vulnerable que no puede cotizar a seguridad social por limitaciones de tipo económico, logre hacerlo a través de un sistema administrado por el Estado, para la obtención de un mínimo vital que garantice la satisfacción de las necesidades básicas del cotizante y su familia.

Asimismo, es importante que el Estado comience a implementar políticas dirigidas a la maximización del beneficio otorgado en los BEPS. Si bien es importante tener en cuenta que las personas que acceden a este mecanismo lo hacen porque no cuentan con los recursos suficientes para acceder a una pensión que, si está planteada desde un salario mínimo vital, debe considerarse el bienestar de estas personas, puesto que al igual que aquellos que acceden al SGP, tienen necesidades fundamentadas en unos costos de vida específicos que deben suplir para vivir dignamente (Ballesteros, et al,. 2014, p. 48).

Partiendo de la base de que los beneficios económicos fueron implementados para incentivar el ahorro individual y permitir a la población más desfavorecida ingresar a un sistema de 
seguridad social, el gobierno colombiano no es lejano a este tipo de transformaciones tributarias e involucra dentro de este régimen especial a las personas naturales que sean elegibles para pertenecer al servicio social complementario de los beneficios económicos periódicos (BEPS), para ser sujetos pasivos del monotributo, mediante la expedición de la Ley 1819 de 2016, así como también la implementación de este en el Registro Único Tributario.

En un primer acercamiento, se pudo identificar que el esfuerzo del Gobierno, para lograr la inscripción masiva al nuevo impuesto del monotributo, fue contraproducente, en el sentido en que los comerciantes consideran que es otra forma más de recaudar dinero para favorecer a unos pocos y con el esfuerzo de la población más vulnerable y, peor aún, con el dinero que muchos hogares necesitan para suplir los requerimientos de vivienda, alimentación, vestuario, educación, entre otros; ya que varios de estos en muchos casos simplemente no se atienden, debido a que su actividad comercial solo es para subsistir y no se cuenta con otro ingreso adicional que les permita mejorar su calidad de vida, obtener estabilidad económica a futuro o procurar una vejez digna.

Al interpretar algunas respuestas, se puede evidenciar que los microempresarios tienen un concepto negativo sobre el uso eficiente del recaudo, ya que para ellos la corrupción es subsidiada a través de los impuestos, motivo por el cual el Estado en su afán de obtener mayores recursos para el déficit que deja dicha corrupción, genera o se «inventa» todo tipo de impuestos «aparentemente» con unos beneficios, pero la realidad que perciben los comerciantes es que estos recursos no son retribuidos a la comunidad, en ninguno de los componentes de vital importancia para la sociedad en general y por el contrario es malgastado o hurtado por unos pocos para solventar las comodidades a las que están acostumbrados.

En consecuencia, a lo anterior, no es posible obtener buenos resultados en la primera y segunda etapa de inscripción al monotributo, toda vez que la mayor parte de los comerciantes consideran que los impuestos no tienen un fin común y por tal razón redoblan sus esfuerzos para no pagar más de lo que consideran justo y así ofrecer sus productos y servicios; motivo por el cual es apenas lógico que los entrevistados tengan un conocimiento mínimo o nulo frente a los temas tributarios. De acuerdo con ello, tres cuartas partes de los entrevistados respondieron que no tenían conocimiento sobre el beneficio financiero del monotributo, es decir, que solo el $7 \%$ tenía conocimiento que a través de este recaudo podía acceder más fácilmente a un servicio financiero y una mínima parte representada en el $3 \%$ respondió algo totalmente irrelevante.

Es importante considerar que los comerciantes manifiestan que la poca o nula acogida a este sistema no está solo ligada a la falta de información, sino al desinterés del Estado en involucrar detalladamente a la comunidad en los temas tributarios, que para el caso que nos atañe hubiese sido verdaderamente positivo las campañas puerta a puerta en los establecimientos, la presencia en los barrios, en las comunas, mediante diferentes actividades pedagógicas que permitieran masificar el tema, resolver inquietudes, resaltar y optimizar los beneficios que ofrece este nuevo sistema de tributación y por último promover la cultura de la contribución voluntaria, que no es más que el principal objetivo del monotributo «un impuesto voluntario».

Los microempresarios no ven la necesidad de inscribirse en un impuesto voluntario, por el contrario lo identifican como una obligación 
más que por ahora pueden ignorar, pero que en algún momento empezarán a tributar cuando el Gobierno decida cambiar su aplicación a «obligatoria», estén ellos de acuerdo o no y quizás sin capacidad para solventarla, pues consideran que hay otros tributos como Cámara de Comercio e Industria y Comercio demasiado onerosos que nos les permiten obtener buenas ganancias para pagar la seguridad social de todos los miembros de su familia y ofrecer mejores oportunidades a sus hijos en educación o simplemente disponer de mayores ingresos para reutilizarlos de la mejor manera posible.

Desde el punto de vista de los microempresarios entrevistados, inscribirse al monotributo no es una opción viable, máxime con tan poca información que se tiene al respecto, sin embargo al cuestionar sobre cuál es el beneficio que escogerían para cubrir dentro del mismo recaudo del monotributo, casi la mitad de los comerciantes eligieron los BEPS, lo que quiere decir que el ideal de la población objeto, es tener una vejez digna o al menos con sus necesidades básicas satisfechas, ya que en consecuencia de sus actividades comerciales informales no podrían ser contribuyentes del sistema general de pensión, e identifican el monotributo como una posibilidad de mejorar sus expectativas frente al tema.

Además, se evidencia claramente que los comerciantes optarían por cubrir un sistema de protección para riesgos de incapacidad, invalidez o muerte, lo que lleva a pensar que al no poder cubrir como sucede en la mayoría de los casos, el sistema general de pensión, desearían ante cualquier calamidad inesperada tener algún tipo de protección para ellos y sus familias. Pero llama la atención, que los comerciantes muestran menor interés por los beneficios existentes con las cajas de compensación familiar, el cual está determinado mediante el artículo 172 de la Ley 1819 del 2016, es decir, que las oportunidades de mejorar su calidad de vida en materia de subsidios de vivienda, recreación y esparcimiento es mínima o simplemente no contemplan esta opción.

Los resultados obtenidos sobre la actitud que asumen los microempresarios en cuanto al cumplimiento de sus obligaciones formales, se pueden identificar cuatro modelos de comportamiento basados en similitudes y diferencias del análisis de contenido realizado a la información recopilada en las entrevistas, clasificados de la siguiente manera:

- Actitud de cumplimiento I: en la cual se encuentran los comerciantes que siempre buscan hacer lo correcto, por lo cual, en todas las respuestas dadas en la entrevista, se evidencia una adecuada organización del negocio y el cumplimiento voluntario de sus obligaciones formales y se encuentran identificados en un $10 \%$ de la población objeto de estudio.

- Actitud de cumplimiento II: en este tipo de comportamiento podemos identificar a los microempresarios que quizás por falta de información o desconocimiento sobre todos los temas tributarios, no logran cumplir con sus cargas impositivas, motivo por el cual en las entrevistas se refleja un porcentaje del $22 \%$ que consideraría inscribirse en el monotributo.

- Actitud de cumplimiento III: en este último comportamiento se encuentran los comerciantes que independientemente tengan o no el conocimiento o la obligación de cumplir ciertos impuestos, no desean cumplir o simplemente han decido no cumplir, por ello utilizan todo tipo de estrategias que les permitan evadir el cumplimento de sus obligaciones formales, los cuales corresponden al $68 \%$ de los entrevistados. 


\section{Conclusiones}

- La poca o nula acogida que tuvo la inscripción al monotributo no está solo ligada a la falta de información, sino al desinterés del Estado en involucrar detalladamente a la comunidad en los temas tributarios, que para el caso que nos atañe hubiese sido verdaderamente positivo las campañas puerta a puerta en los establecimientos, la presencia en los barrios, en las comunas, mediante diferentes actividades pedagógicas que permita masificar el tema, resolver inquietudes, resaltar y optimizar los beneficios que ofrece este nuevo sistema de tributación.

La opción más viable en materia de beneficio asociado al monotributo son los beneficios económicos periódicos (BEPS) como servicio social complementario, que indica un ideal de la población objeto de tener una vejez digna o al menos con sus necesidades básicas satisfechas, ya que en consecuencia de sus actividades comerciales informales no pueden ser contribuyentes del Sistema General de Pensión e identifican el monotributo como una posibilidad de mejorar sus expectativas frente al tema.

El artículo 905 del Estatuto Tributario indica las condiciones que se debe reunir para ser monotributistas, sin embargo, el numeral 2 de la citada norma indica que la actividad económica se debe desarrollar en un área igual o inferior a 50 metros cuadrados, lo que discrepa con el Grupo 479 de la División 47 comercio al por menor, definidas en el numeral 4 de la misma norma, y corresponde a Comercio al por menor no realizado en puestos de venta, establecimientos o mercados, respectivamente.
- Existe un grado alto de desinformación por parte de los comerciantes del sector de Minitas, La Cumbre y Viveros, sobre las obligaciones tributarias, quién administra los impuestos, qué es un impuesto y, además, para qué sirve, fundamentalmente con respecto al impuesto del monotributo, a razón de que no cuentan con la cultura tributaria y mucho menos con la responsabilidad como comerciantes de contribuir a la formalidad en el país.

Los beneficios económicos periódicos son considerados por los entrevistados como la opción más atractiva, lo que establece en primera instancia que existe alguna intención del comerciante en mejorar su calidad de vida y buscar una estabilidad económica, pensando en el futuro tanto para ellos como para sus familias.

Se detecta la necesidad de realizar por parte de la administración tributaria capacitaciones que orienten de forma clara y precisa a los comerciantes sobre los requerimientos para ser parte del esquema del monotributo y las obligaciones que contraerán.

- La mayor parte de la población entrevistada, comprendida por los comerciantes de los barrios La Cumbre, Viveros y Minitas, no contemplan en un corto plazo inscribirse en el monotributo de manera voluntaria, principalmente por las cargas tributarias onerosas que en el momento poseen y por falta de información sobre el mismo impuesto y sobre los beneficios asociados a este nuevo tributo opcional. 


\section{Referencias}

- Ballesteros, A., Herrera, D. F \& Parra, P. A. (2014). ¿Beneficios económicos periódicos: ¿Una alternativa para la vejez digna de los más vulnerables? (Bachelor's thesis, Universidad Libre). Recuperado de http://repository.unilibre. edu.co/handle/10901/7690?locale-attribute=es

- Bonilla, E. C. (2014). La cultura tributaria como herramienta de política fiscal: la experiencia de Bogotá. Revista Ciudad. Estados Política, 1(1), 21-35.

- Decreto 2640 (2013). Por el cual se reglamenta el artículo 555-2 de Estatuto Tributario. Recuperado de http://www.alcaldiabogota.gov.co/sisjur/normas/Norma1.jsp?i=59604

- Departamento Administrativo Nacional de Estadística. (2012). Clasificación de Actividades Económicas Revisión 4 adaptada para Colombia CIIU Rev. 4 A.C. Recuperado de http://www.dian.gov.co/descargas/normatividad/2012/Resoluciones/CIIU_Rev4ac.pdf

- Departamento Administrativo Nacional de Estadística. (2017). Empleo Informal y Seguridad Social. Recuperado de http://www.dane.gov.co/index.php/estadisticas-por-tema/mercado-laboral/ empleo-informal-y-seguridad-social

- Dirección de Impuestos y Aduanas Nacionales. (2017). Estatuto Tributario. Recuperado de http://www.dian.gov.co/dian/15servicios.nsf/etributario?OpenView\&Start $=1$ \& Count $=1000 \&$ Expand $=9 \# 9$
- Encolombia. (2017). Guía para el Trámite de Creación y Formalización de Una Empresa. Recuperado de https://encolombia.com/economia/economiacolombiana/emprendimiento/ guiaparaeltramitedecreacion/2/

Galvis, K. L. (2015). Asesoría en los trámites a toda persona natural o jurídica para el cumplimiento de sus obligaciones administradas ante la dirección de impuestos $\mathrm{y}$ adunas nacionales DIAN punto de contacto Ocaña. Recuperado de http://repositorio.ufpso.edu.co:8080/dspaceufpso/ bitstream/123456789/726/1/27853.pdf

González, D. (2006). Regímenes Especiales De Tributación Para Pequeños Contribuyentes En América Latina. Banco Interamericano De Desarrollo. Recuperado de https:// publications.iadb.org/handle/11319/2302

- Ley 100. (1993). Por la cual se crea el sistema de seguridad social integral y se dictan otras disposiciones. Recuperado de http:// www.secretariasenado.gov.co/senado/basedoc/ley_0100_1993.html

Ley 1819. (2016). Por medio de la cual se adopta una reforma tributaria estructural, se fortalecen los mecanismos para la lucha contra la evasión y la elusión fiscal, y se dictan otras disposiciones. Recuperado de http://es.presidencia.gov.co/normativa/ normativa/LEY\%201819\%20DEL\%2029\%20 DE\%20DICIEMBRE\%20DE\%202016.pdf

Mérida, J. A. (2014). Regulación de los negocios del microempresario informal para su inserción en el marco legal fiscal vigente en Guatemala. Recuperado de http://www.repositorio.usac.edu.gt/1279/1/03_4785.pdf 
- Organización Internacional del Trabajo. (2014). Monotributo en América Latina. Recuperado de http://www.ilo.org/wcmsp5/ groups/public/---americas/---ro-lima/documents/publication/wcms_357452.pdf

- Ortiz, D.C. (2016). Análisis de las experiencias en Argentina y Perú de formalización laboral como referentes para la evaluación y diseño de una política pública en Colombia: periodo 2000-2010. Recuperado de http://repositorio.urosario.edu.co/ handle/10336/12949

- Rodríguez, J.M., Chaumont M.L \& León, T.C. (2011). Monotributo: impacto de los regímenes desde su nacimiento hasta la actualidad. Recuperado de http://m.bdigital. uncu.edu.ar/objetos_digitales/4742/tesinarodriguez-chaumon.pdf

- Rojas, H. L. (2015). Análisis de la ética empresarial en las importancias: un enfoque desde el subsector de las confecciones en Colombia para los años 2013 y 2014. Recuperado de https://repository.unimilitar.edu. co/bitstream/10654/7599/1/RojasCasta\%C3\%B1 edaHaroldLibardo2015.pdf

- Vega, C. (2015). El espectador. Colombia no tiene cultura tributaria. Recuperado de https://www.elespectador.com/noticias/ economia/colombia-no-tiene-cultura-tributaria-articulo-543559

\section{Bibliografía complementaria}

- Acuerdo 589. (2004). Por medio del cual se establece la división de municipio de Manizales en comunas y corregimientos. Recuperado de http://www.manizales.gov.co/ RecursosAlcaldia/201611281243527274.pdf

- Constitución Política de Colombia. (1991). Diario Oficial de la república de Colombia, Bogotá, No. 116 del 20 de julio de 1991. Recuperado de http://www.secretariasenado. gov.co/senado/basedoc/constitucion_politica_1991.html

- Decreto 604. (2013). Por el cual se reglamenta el acceso y operación del servicio social complementario de beneficios económicos periódicos (BEPS). Recuperado de http://www.alcaldiabogota.gov.co/sisjur/ normas/Norma1.jsp?i=52482

- Decreto 738. (2017). Por el cual se adiciona el epígrafe de la parte 5 y un título a la parte 5 del libro 1 y se adicionan y modifican literales, incisos y artículos del título 1 de la parte 6 del libro 1 del decreto 1625 de 2016 Único Reglamentario en Materia Tributaria para reglamentar el monotributo. Recuperado de http://www.comunidadcontable. com/BancoMedios/Documentos\%20PDF/ decreto $\% 20738 \% 20$ del $\% 2008 \% 20$ de $\% 20$ mayo\%20de\%202017.pdf

- Ley 1151. (2007). Por la cual se expide el Plan Nacional de Desarrollo 2006-2010. Recuperado de http://www.alcaldiabogota. gov.co/sisjur/normas/Norma1.jsp?i=25932 
- Ley 1562. (2012). Por la cual se modifica el sistema de riesgos laborales y se dictan otras disposiciones en materia de salud ocupacional. Recuperado de http://www. alcaldiabogota.gov.co/sisjur/normas/Norma1.jsp?i=48365

- Ley 789. (2002). Por la cual se dictan normas para apoyar el empleo y ampliar la protección social y se modifican algunos artículos del código sustantivo de trabajo. Recuperado de http://www.alcaldiabogota. gov.co/sisjur/normas/Norma1.jsp?i=6778
- Ley 1429. (2010). Por la cual se expide la ley de formalización y generación de empleo. Recuperado de http://www.secretariasenado.gov.co/senado/basedoc/ ley_1429_2010.html

- Ley 1607. (2012). Por la cual se expiden normas en materia tributaria y se dictan otras disposiciones. Recuperado de https:// www.icbf.gov.co/cargues/avance/docs/ ley_1607_2012.htm 